\title{
Der raumwirtschaftliche Ansatz in der Geographie
}

\author{
Zum Band: «Wirtschaftsgeographie 1» von Ludwig Schätzl*
}

(1) Die Abkehr vom Naturdeterminismus in den ersten Jahrzehnten dieses Jahrhunderts ist für die ENTWICKLUNG DER WIRTSCHAFTSGEOGRAPHISCHEN FORSCHUNG von entscheidender Bedeutung. Betrachtungsweisen, die von einer einseitigen Bestimmung menschlichen Handelns durch die naturräumlichen Gegebenheiten ausgehen, verlieren an Aktualität. Ins Zentrum rückt statt dessen immer mehr die Erklärung der räumlichen Ordnung der Wirtschaft als Ergebnis von Entscheidungen der wirtschaftenden Menschen. Gleichzeitig vollzieht sich eine inhaltliche Veränderung des Landschaftsbegriffes. Die Wirtschaftsgeographie beschränkt sich nicht mehr auf die Beschreibung physiognomisch fassbarer Elemente der Landschaft. In zunehmendem Masse wendet sie sich der Analyse funktionaler Strukturen zu. Der Wirtschaftsraum wird schliesslich als interdependentes System aufgefasst.

Mit dieser Entwicklung ist eine zunächst allerdings noch zögernde Hinwendung zu mode11-theoretischen Fragestellungen verbunden. Die Kontakte zu den benachbarten, eher theoretisch ausgerichteten Wirtschafts- und Sozialwissenschaften intensivieren sich. Die Wirtschaftsgeographie bleibt zwar vorwiegend empirisch ausgerichtet, die Anwendung raumanalytischer Methoden der Wirtschafts- und Sozialwissenschaften führen in der Folge aber doch zu einer Reihe von eigenständigen Beiträgen zur Erstellung einer Theorie der räumlichen Ordnung der Wirtschaft. Die in neuerer Zeit noch enger werdenden Kontakte sind durch ein immer stärkeres Bedürfnis nach einer einheitlichen und fundierten theoretischen und methodischen Grundlegung für die Wirtschaftsgeographie bedingt.

McNee (1959), Chisholm (1966) und Bartels (1970) haben parallel zu diesen Anstrengungen notwendige Beiträge zur Klärung der spezifischen Optik und des eigenen disziplingeschichtlichen. Standorts der Wirtschaftsgeographie innerhalb der wirtschafts- und sozialwissenschaftlichen Disziplinen geliefert.
(2) Im vorliegenden Band ist Schätzl - wie vor ihm zum Beispiel schon Boesch (1977) - der Auffassung, dass sich der Forschungsgegenstand der Wirtschaftsgeographie auf die Gesamtheit der wirtschaftlichen Aktivitäten erstrecke und sich keinesfalls auf die in der Landschaft sichtbar werdenden Aspekte beschränke. Entsprechend definiert er Wirtschaftsgeographie - und er unschreibt damit ihre potentiellen Forschungsfelder - relativ umfassend als die Wissenschaft von der räumlichen Ordnung und räumlichen Organisation der Wirtschaft. Sie stellt sich unter dem von Schätzl schon 1974 inhaltlich skizzierten und nun präszisierten RAUMWIRTSCHAFTLICHEN ANSATZ die Aufgabe, "räumliche Strukturen und ihre Veränderungen - aufgrund interner Entwicklungsdeterminanten und räumlicher Interaktionen - zu erklären, zu beschreiben und zu bewerten" (S. 16). Die Verteilung wirtschaftlicher Aktivitäten im Raum (Struktur), die räumlichen Interaktionen (Wirkungszusammenhänge) zwischen den wirtschaftlichen Aktivitäten (Funktion) sowie deren Entwicklungsdynamik (Prozess) sind dabei als interdependentes Raumsystem zu verstehen. In der Konzeption Schätzls setzt sich die wirtschaftsgeographische Wissenschaft aus den Teilbereichen Theorie, Empirie und Politik zusammen, die in enger Wechselbeziehung zueinander stehen. Diese Betrachtungsweise, die sich in vielen Nachbarwissenschaften längst bewährt hat, ist auch in der Wirtschaftsgeographie nicht neu. Als Zeichen jüngster Entwicklung ist allerdings das Gewicht, das den Teilbereichen Theorie und Politik beigemessen wird, zu werten.

Wenn auch die Methodologie der Wirtschaftsgeographie, die Formulierung expliziter Hypothesen und die Qualität des verfügbaren methodischen Instrumentariums in den letzten Jahren entscheidend verbessert wurden, so ist Schätzl

Christian Hanser, dipl. Geogr., Geographisches Institut der Universität Zürich

Walter H. Rambousek, Dr. phil., London School of Economics and Political Science

* Ludwig Schätzl, Wirtschaftsgeographie 1: Theorie, UTB No. 782, Ferdinand Schöningh, Paderborn 1978,175 S., DM 14.80 
nicht allein, wenn er darauf hinweist, dass trotz erwähnter Anstrengungen die theoretische Erklärung der räumlichen Ordnung der Wirtschaft in der Wirtschaftsgeographie bisher vernachlässigt wurde (vgl. Bahrenberg, 1979; Bartels 1970). In den deutschsprachigen Lehrbüchern wurden in der Regel nur die weniger komplexen Raumwirtschaftsmodelle wie jene von Thünen, Christaller oder Fourastié eingehender behandelt (vg1. Boesch, 1977; Otremba 1969). Im Gegensatz dazu sei auf die englischsprachige Literatur hingewiesen, wo Abler/Adams/Gould (1971), Haggett (1965) oder Smith (1971) schon vor geraumer Zeit in ihren Lehrbüchern ganze Paletten raumwirtschaftlicher Modelle vorstellten.

Auch wenn die bislang vor allem von Oekonomen entwickelten Theorien und Modelle wegen ihres hohen Abstraktionsgrades zur Zeit nur bedingt operationabe1 sind, sieht Schätzl doch ihre zunehmende Bedeutung für wirtschaftsgeographische Fragestellungen. Bei realistischer Einschätzung der Interessen und des Forschungspotentials geht jedoch auch Schätzl davon aus, dass die Empirie wie in Vergangenheit und Gegenwart auch in der Zukunft das Schwergewicht wirtschaftsgeographischer Forschung darstellen wird. Er betont deshalb auch die grosse Bedeutung, die der Erfassung, Beschreibung und Analyse räumlicher Strukturen, Funktionen und Prozesse (empirische Regionalanalyse) beizumessen sei. Er sieht aber auch die wichtigkeit empirischer Arbeiten für die Theoriebildung, die Lösung aktueller Regionalprobleme und die Prognose und Gestaltung künftiger Prozessabläufe. Empirisch überprüfte Modelle der räumlichen Ordnung und Organisation der Wirtschaft dienen als Entscheidungshilfe für die Regionalpolitik. Der Wirtschaftsgeographie wird damit neben der Theoriebildung und der empirischen Regionalanalyse auch die Aufgabe zugewiesen, einen Beitrag zur Lenkung des räumlichen Prozessablaufs in Richtung auf eine Optimierung wirtschaftlicher und gesellschaftlicher Zielsetzungen zu leisten (Regionalpolitik). Schätzl erachtet diesen wissenschaftlichen Teilbereich als ebenso wichtig.

Mit dieser Konzeption weist der Autor der Wirtschaftsgeographie nicht etwa eine Zubringerfunktion für nahestehende Sozialwissenschaften zu, vielmehr glaubt er mit dieser Konzeption den zentralen Aufgaben der Wirtschaftsgeographie am ehesten gerecht zu werden. Er verfolgt damit also nicht das Ziel, die Abhängigkeit der Wirtschaftsgeographie von den Nachbarfächern $z u$ vergrössern, sondern ihr im Gegenteil die Grundlagen bereitzustellen, mit denen sie imstande ist, eigenständige Leistungen $\mathrm{zu}$ vollbringen.

Allerdings sind geographische Hochschulinstitute, die sich der Erarbeitung von Grundlagen für die Regionalpolitik widmen, nach wie vor schwer zu finden. Dafür schlossen sich die an Raumpolitik interessierten Vertreter verschie- denster Disziplinen unter dem Nachfragedruck entsprechender politischer Entscheidungsträger schon frühzeitig in eigenen Institutionen zusammen (zum Beispiel Regional Science Association). Seit wenigen Jahren gewannen dennoch eine Reihe von Wirtschaftsgeographen an Universitäten mit einer auf praktische Probleme der Regionalpolitik ausgerichteten Forschungstätigkeit an Gewicht. Im Zentrum ihrer Arbeiten standen in der Regel Analysen von Verdichtungsund Entleerungsräumen in Industrie- und Entwicklungsländern.

(3) Da die Verteilung wirtschaftlicher Tätigkeiten im Raum, die Verflechtungsbeziehungen zwischen den Standorten sowie die Veränderung der Raumstruktur als interdependentes Raumsystem verstanden werden, gliedert Schätzl sein LEHRBUCH 'WIRTSCHAFTSGEOGRAPHIE 1 ' nicht nach dem traditionellen Sektorprinzip, das der partialanalytischen Betrachtungsweise einer als Produktions- und Handelsgeographie verstandenen Wirtschaftsgeographie entgegenkommt. Vielmehr wählt der Autor - gemäss seinem Konzept - eine Gliederung in die Teilbereiche Theorie, Empirie und Regionalpolitik. Der hier zu besprechende erste Band befasst sich mit der Theorie der räumlichen Ordnung und der räumlichen Organisation der Wirtschaft. Nach bewährtem Muster nimmt der Autor dabei eine Zweiteilung vor, wobei zuerst die Standorttheorien und in einem zweiten Teil die regionalen Wachstums- und Entwicklungstheorien behande1t werden. Aus der Vielzahl bisher entwickelter theoretischer Erklärungsversuche wirtschaftlicher Raumsysteme werden diejenigen berücksichtigt, die in der raumwissenschaftlich-wirtschaftsgeographischen Diskussion als grundlegend gelten und zudem die wesentlichsten langfristigen Entwicklungstendenzen der Theoriebildung aufzuzeigen vermögen.

Die Behandlung der Standorttheorien erfolgt anhand der üblichen Unterteilung in einzelwirtschaftliche Theorien der unternehmerischen Standortwah1 (Industriestandorttheorie A. Webers und deren Weiterentwicklung durch W. Isard und D.M. Smith) und in gesamtwirtschaftliche Standorttheorien (Theorie der Landnutzung, J.H. von Thünen; Theorie der zentralen Orte, W. Christaller; Theorie der Marktnetze, A. Lösch; sowie die Integration der Standortstrukturtheorien und Weiterentwicklung der partialtheoretischen Ansätze zu einer allgemeinen Theorie des räumlichen Gleichgewichts, der sogenannten Raumwirtschaftstheorie, durch W. Isard und E. von Böventer).

Bei der Vorstellung des viel heterogeneren Theorienbündels der regionalen Wachstums- und Entwicklungstheorien, die von monokausalen Erklärungsversuchen bis zu komplexen Theorieansätzen reichen, verfolgt Schätzl das Ziel, die ganze Breite des Spektrums unterschiedlicher Erklärungsmöglichkeiten regionalen Wirtschaftswachstums auszuleuchten. In ihrem Ergebnis lassen 
die Wachstums- und Entwicklungstheorien drei Richtungen erkennen: Theorien, die einen interregionalen Ausgleich des Wohlstands erwarten; Theorien, die von einer zunehmenden Verschärfung der wirtschaftlichen und sozialen Ungleichgewichte ausgehen und schliesslich Theorien, welche die Frage nach gleichgewichtiger versus ungleichgewichtiger Entwicklung in Abhängigkeit vom Entwicklungsstand einer Volkswirtschaft beantworten. Diesen Tatbestand nimmt Schätzl als Ausgangspunkt für die Behandlung verschiedener Theorieansätze, die er in entsprechender Reihenfolge vorstellt: die neoklassische Theorie, die postkeynesianische Theorie (u.a. E.D. Domar und R.F. Harrod, A.O. Hirschman), die Exportbasis-Theorie (J.S. Duesenberry, D.C. North), die Wirtschaftsstufentheorie (H. Bobek, J. Fourastié, W.W. Rostow) sowie polarisationstheoretische Ansätze. Bei der Diskussion des letzten Komplexes wird zwischen sektoraler (F. Perroux, J.Schumpeter) und regionaler (G.Myrdal, A.O. Hirschman) Polarisation unterschieden. Als Weiterentwicklungen des polarisationstheoretischen Ansatzes werden Wachstumspolkonzepte (J.R. Boudeville, J. Paelinck) und Zentrum-PeripherieMode1le (J. Friedmann, R. Prebisch) vorgestellt. Berücksichtigt werden schliesslich auch innovationstheoretische Ansätze (J.R. Lasuén).

Zum Schluss werden die ersten Integrationsversuche von Standort theorien und regionaler Wachstums- und Entwicklungstheorien behandelt (E. von Böventer, H.W. Richardson).

Wenn wir versuchen, Schätzls Buch im Vergleich mit bisherigen deutschsprachigen Lehrbüchern $\mathrm{zu}$ bewerten, so wollen wir festhalten, dass damit kein Ersatz für bestehende wirtschaftsgeographische Einführungen geschaffen ist. Es wird auch keine neue Wirtschaftsgeographie angeboten. Allerdings hebt sich die Optik, durch welche ein raumwirtschaftliches Gefüge betrachtet wird, von traditionellen Beschreibungen handels- oder produktionsgeographischer Wirtschaftsräume ab. Mit der Berücksichtigung aktueller Entwicklungen und dem Einbezug bisher vernachlässigter Aspekte des Faches, erfolgt zwangsläufig eine stärkere Ausrichtung auf die Regionalforschung.

Standorttheorien sind schon in anderen Lehrbüchern zusammengestellt worden. Neu ist vielmehr die konzentrierte Behandlung regionaler Wachstums- und Entwicklungstheorien. Besonders gelungen scheint uns die unfangmässige Gestaltung der einzelnen Abschnitte. Theorien werden nicht auf einer oder zwei Seiten abgetan und die einzelnen Abschnitte zu jeweils etwa zehn Seiten sind trotzdem nicht so lang, dass der Leser den roten Faden und sich selbst in Detailinformationen verliert.

Mit dem Instrumentarium, das uns der Autor vorstellt, ermöglicht er problemorientierte wirtschaftsgeographische Arbeiten, die sich am Wissensstand der benachbarten Regionalwissenschaften ausrichten. Auf die grosse Bedeutung regio- nalwissenschaftlicher Kenntnisse für Arbeiten in der Wirtschaftsgeographie weist zuletzt Bahrenberg (1979) hin. Nur bei Beherrschung dieser Grundlagen sei die Wirtschaftsgeographie fähig in Problembereichen wie der Analyse und Veränderung internationaler und interregionaler Ungleichgewichte problem- und politikorientiert mitzuarbeiten und gleichzeitig auch theoretisch fundierte Arbeiten zu liefern. Eine wirtschaftsgeographische Beteiligung an aktueller Problemverarbeitung wird in dieser Richtung von namhaften Autoren immer wieder gefordert (Bahrenberg, 1979; Coppock, 1976; Schätz1, 1974; Smith, 1977) Auf dem Weg dahin, wird das vorliegende Buch ohne Zweifel Schrittmacherdienste leisten.

Schätzls Buch wendet sich an Anfänger. Eine klare Schreibweise, ein transparenter Aufbau, und die hier umrissene klare Vorstellung was Geographie ist, welche Aufgabe sie zu erfüllen hat und erfüllen kann, machen diesen Band sehr lesenswert. 'Termini technici' sind sparsam verwendet, werden aber dort eingeführt, wo es notwendig erscheint, den begrifflichen Sonderzug der Geographie zu verlassen um damit interdisziplinäre Arbeit zu erleichtern. Die abstrakte Formelschreibweise wird im Gegensatz zu vielen Lehrbüchern der Regionalökonomie massvoll verwendet, was den geradlinigen Aufbau unterstützt. Somit ist das Buch auch im Selbststudium zu erarbeiten und eignet sich - nicht zuletzt des günstigen Preises wegen - ganz besonders als Begleitlektüre für Vorlesungen und Seminare. Gute, selektive Literaturhinweise und eine Würdigung der Leistungen verschiedener Autoren erleichtern das Studium weiterführender Literatur.

Schätzl verfolgt mit seinem Buch das sicherlich nicht bescheidene $\mathrm{Zie1}$, einen möglichen, wenn auch keineswegs anspruchslosen Weg aufzuzeigen, um die Wettbewerbsfähigkeit der geographischen Wissenschaft gegenüber den Nachbardisziplinen auch in Zukunft zu erhalten. Gegenwärtige Bedenken im Zusammenhang mit zunehmenden Schwierigkeiten des Schulfaches Geographie und den Erfahrungen im Rahmen des Nationalen Forschungsprogranmes 'Regionalprobleme in der Schweiz' sind wohl durchaus angebracht. Viele Autoren glauben gar, dass die Wirtschaftsgeographie bei stärkerer Hinwendung zu den Regionalwissenschaften endlich ihr langgesuchtes Paradigma finden wird.

Allerdings sind wir mit Bahrenberg der Meinung, dass die Bedeutung der geographischen Wissenschaft nur verstärkt werden kann, wenn sie zu den hier skizzierten Problemen engagiert ihren Teil zu leisten gedenkt (1979, S. 65:'Geographie wird sein, was wir tun werden'.) Und diese Kompetenz wird ohne die wichtigsten Kenntnisse der Oekonomie, Politologie, Soziologie, Ethnologie und Mathematik nicht möglich sein, genau so, wie Kenntnisse der Chemie, Physik oder der Paläobotanik in der Morphologie oder Klimatologie schon längst nicht mehr wegzudenken sind. Mit Spannung darf man auf den Band 'Wirtschaftsgeographie 2' 
warten, von dem wir uns eine Verringerung der in der täglichen Forschungsarbeit oft schmerzlich empfundenen Kluft zwischen Theorie und Empirie erhoffen.

\section{Literaturhinweise:}

- Abler R., J.S. Adams, P. Gould, 1971, Spatial Organization. The Geographer's View of the World, Prentice-Hall, Englewood Cliffs, N.J.

- Bahrenberg G., 1979, Von der Anthropogeographie zur Regionalforschung - eine Zwischenbilanz, S. 59-68, in: Zur Situation der deutschen Geographie zehn Jahre nach Kiel, Osnabrücker Studien zur Geographie, Band 2, Osnabrück, 1979

- Bartels D., 1970, Einleitung, S. 13-45, in: Wirtschafts- und Sozialgeographie, hrsg. von Dietrich Bartels, Neue Wissenschaftliche Bibliothek 35, Wirtschaftswissenschaften, Kiepenheuer \& Witsch, Köln, Berlin

- Boesch H., 1977, Weltwirtschaftsgeographie, Westermann, Braunschweig, 4. Auflage

- Chisholm M., 1966, Geography and Economics, G. Be11 \& Sons, London
- Coppock J.T., 1976, Geography and Public Policy: Challenge, Opportunity and Implications, Chapter 1, p. 1-19, in: Spatial Dimensions of Public Policy, ed. by J.T. Coppock and W.R.D. Sewe11, Oxford

- Haggett P., 1965, Locational Analysis in Human Geography, Edward Arnold, London

- McNee R., 1959, The Changing Relationship of Economics and Economic Geography, p. 189-198, in: Economic Geography, Volume 35

- Otremba E., 1969, Die Güterproduktion im Weltwirtschaftsraum, Stuttgart, 2. Auflage

- Schätz1 L., 1974, Zur Konzeption der Wirtschaftsgeographie, Akademische Antrittsvorlesung, Universität Giessen, 28.1.1974, S.124134, in: Die Erde, 105.1974, 2

- Smith D.M., 1971, Industrial Location: An Economic Geographical Analysis, John Wiley, New York u.a.

- Smith D.M., 1977, Human Geography, A Welfare Approach, Edward Arnold, London

\section{I TERATURBESPRECHUNGEN}

MESSERLI, Bruno / AERNI, Klaus: Carthography and its Application for Geographical and Ecological Problems. Simen Mountains - Ethiopia. Vol.1, Geographica Bernensia G 8, Beiheft 5 zum Jahrbuch der Geographischen Gesellschaft von Bern, $102 \mathrm{~S}$, 34 Abb., Tab., Schriftt., Karten, Selbstverlag des Geographischen Instituts der Universität Bern, 1978. Fr. 36.--

Dieses Beiheft vermittelt eine vorzügliche Idee von einer modernen Form der geographischen Erschliessung einer Region, hier des Hochlandes und Hochgebirges Semien zwischen Tanasee und Asmara. In der Zeit zwischen 1968 und dem politischen Umschwung 1976 arbeitete ein grösseres interdisziplinäres Team von Berner und Zürcher Wissenschaftern in diesem Gebiet an ökologischen Fragen. Um diese sich über mehrere Jahre erstrekkenden Untersuchungen auf eine solide Basis zu stellen, entschloss man sich, auf der Grundlage von amerikanischen Luftbildern und eigenen Vermessungen und Felderhebungen eine topographische Karte 1: 25'000 zu erstellen. $370 \mathrm{~km}^{2}$ wurden photogrammetrisch ausgewertet und in der Darstellungsart des einfarbigen schweizerischen Uebersichtsplanes 2 eindrückliche Kartenblätter gestaltet. Die mannigfachen Probleme bei ihrer Erstellung, nicht zuletzt auch die Schwierigkeiten mit der Nomenklatur, werden im Bericht von P. Stähli und M. Zurbuchen eingehend und gut illustriert erläutert. In drei Beiträgen wird zudem die Verwendung dieser Basiskarten für Detail- untersuchungen und Kartierungen beschrieben. Für seine Studien über die Veränderungen der Siedlungen kombinierte P. Stähli terrestrische Aufnahmen von $1954 \mathrm{mit}$ den vorhandenen Luftbildern und eigenen Aufnahmen im Jahre 1975. Die Ergebnisse sind in einem mehrfarbigen thematischen Aufdruck und weitern Kartenskizzen festgehalten. Im Text finden sich ausführliche Angaben über die Bevölkerung, Besiedlung und Kulturlandgewinnung, sowie über die Interessenkonflikte im Bereich des Nationalparkes. Auf derselben Kartengrundlage wurden von $\mathrm{H}$. Hurni die verschiedenen Formen der Bodenerosion kartiert, welche vor allem das steile Ackerland unfruchtbar machen. Mit dieser Untersuchung wurde für die ins Auge zu fassenden Schutzmassnahmen eine klare Ausgangslage geschaffen. Von R. Nägeli stammen zwei grossmassstäbliche Kartenbeilagen des Marktfleckens Debark, ein detailliertes Inventar der funktionalen Gliederung sowie der baulichen Entwicklung dieser Stadt in den Jahren 1964-76. Der Begleittext dazu vermittelt ein anschauliches Bild eines aethiopischen Gewerbe- und Handelszentrums. Alle Beiträge sind in Englisch abgefasst, mit deutschen und französischen Zusammenfassungen und ausführlichen Literaturhinweisen versehen. Diese erstaunliche, mit grossem Aufwand und Einsatz geleistete Grundlagenbeschaffung und geographische Arbeit verdient ein breites Interesse.

GH $1980 / 2$

Ernst Spiess, Zürich 